ERRATUM

\title{
Anti-Müllerian hormone confirms the novel classification of female functional androgenization including polycystic ovary syndrome
}

B Wetzka, W Textor, A Ochsner and F Geisthövel

Centre for Endocrinology and Reproductive Medicine Freiburg (CERF), Bismarckalle 7f, D-79098 Freiburg im Breisgau, Germany

The journal and the authors apologise for errors in the above article published in the August issue of this journal, vol 165 pp 323-330. The errors occur in Tables 1 and 2. In Table 1, the cut-off value for 17-OH-P should be $>30$ and in Table 2, the value for $2 \mathrm{~h}$ glucose for FAS II should be $4.9 \pm 0.98$ and not as published. Both these tables are corrected and published in full below.

Table $1 \mathrm{AMH}$ and primary variables $(2,3)$ for all patients with functional androgenization (FA) compared with the control group. Data are shown as the mean \pm s.D. Upper/lower cut-off levels for FA diagnosis were reviewed in Geisthövel et al. (2).

\begin{tabular}{|c|c|c|c|c|}
\hline Variable & Cut-off value & FA & Control & $\boldsymbol{P}^{*}$ \\
\hline Number of patients & & 178 & 30 & \\
\hline Age (years) & & $25.5 \pm 5.4$ & $30.3 \pm 5.2$ & $<0.0001$ \\
\hline $\mathrm{AMH}(\mathrm{ng} / \mathrm{ml})$ & TBD & $11.1 \pm 6.7$ & $3.0 \pm 2.0$ & $<0.0001$ \\
\hline $\mathrm{LH}(\mathrm{U} / \mathrm{l})$ & $>8.4$ & $10.6 \pm 5.4$ & $5.4 \pm 2.1$ & $<0.0001$ \\
\hline LH/FSH ratio & $>1.3$ & $2.19 \pm 1.12$ & $0.96 \pm 0.39$ & $<0.0001$ \\
\hline Testosterone $(\mathrm{nmol} / \mathrm{l})$ & $>2.1$ & $2.58 \pm 1.0$ & $1.37 \pm 0.4$ & $<0.0001$ \\
\hline SHBG $(\mathrm{nmol} / \mathrm{l})$ & $<25$ & $30.4 \pm 18$ & $51.1 \pm 14.4$ & $<0.0001$ \\
\hline $\mathrm{FAI}$ (testosterone/SHBG $\times 100$ ) & $>5$ & $11.2 \pm 7.9$ & $2.9 \pm 1.3$ & $<0.0001$ \\
\hline DHEAS $(\mu \mathrm{mol} / \mathrm{l})$ & $>9.6$ & $6.71 \pm 3.2$ & $3.95 \pm 1.61$ & $<0.0001$ \\
\hline $17-\mathrm{OH}-\mathrm{P}(\mathrm{nmol} / \mathrm{l})$ & $>30$ & & & \\
\hline $\mathrm{Oh}$ & & $5.63 \pm 14.2$ & $2.67 \pm 1.06$ & $<0.0001$ \\
\hline $1 \mathrm{~h}$ & & $10.6 \pm 25.1$ & $5.67 \pm 1.85$ & $<0.0001$ \\
\hline $\operatorname{EPO}(n \%)$ & - & 69 & 20 & $<0.0001$ \\
\hline \multicolumn{5}{|l|}{ Glucose $(\mathrm{mmol} / \mathrm{l})$} \\
\hline $\mathrm{Oh}$ & $>5.5$ & $4.8 \pm 0.6$ & $4.5 \pm 0.3$ & 0.0085 \\
\hline $1 \mathrm{~h}$ & $>7.7$ & $6.5 \pm 2.2$ & $3.5 \pm 0.7$ & $<0.0001$ \\
\hline $2 \mathrm{~h}$ & & $5.2 \pm 1.6$ & $4.7 \pm 1.2$ & 0.0005 \\
\hline \multicolumn{5}{|l|}{ Insulin (mU/I) } \\
\hline $\mathrm{Oh}$ & $>14.4$ & $11 \pm 12$ & $5.2 \pm 2.8$ & $<0.0001$ \\
\hline $1 \mathrm{~h}$ & $>97.8$ & $98 \pm 89$ & $31.2 \pm 18.3$ & $<0.0001$ \\
\hline $2 \mathrm{~h}$ & & $81 \pm 115$ & $26.7 \pm 15.1$ & $<0.0001$ \\
\hline $\mathrm{BMI}\left(\mathrm{kg} / \mathrm{m}^{2}\right)$ & $>24$ & $27.0 \pm 6.5$ & $21.1 \pm 1.8$ & $<0.0001$ \\
\hline
\end{tabular}

$\mathrm{AMH}$, anti-Müllerian hormone; SHBG, sex hormone-binding globulin; FAI, free androgen index; 17-OH-P, 17-hydroxy-progesterone; BMI, body mass index; TBD, to be determined. *The $P$ value was derived from Wilcoxon rank sum test. To compare the presence of enlarged polyfollicular ovaries (EPO), Fisher's two-sided exact test was used. 
Table 2 Variables with significant differences between the FA groups and controls. All data are shown as the mean \pm s.D.

\begin{tabular}{|c|c|c|c|c|c|c|}
\hline Variable & FAS I & FAS II & FAS III & FAS IV & FCA & Control \\
\hline$n$ & 51 & 27 & 74 & 16 & 11 & 30 \\
\hline Age (years) & $25.6 \pm 3.9$ & $25.5 \pm 7.0$ & $26.2 \pm 5.7$ & $22.1 \pm 4.4$ & $24.5 \pm 5.7$ & $30.3 \pm 5.2$ \\
\hline $\mathrm{AMH}(\mathrm{ng} / \mathrm{ml})$ & $15.6 \pm 5.8(1 \mathrm{a}-\mathrm{e})$ & $5.05 \pm 3.01(2 b, c)$ & $11.6 \pm 6.6(3 c-e)$ & $6.9 \pm 4.6(4 c)$ & $7.0 \pm 3.8(5)$ & $3.0 \pm 2.0$ \\
\hline $\mathrm{LH}(\mathrm{U} / \mathrm{l})$ & $14.0 \pm 4.9(1 \mathrm{a}-\mathrm{e})$ & $5.7 \pm 3.4(2 b)$ & $11.3 \pm 4.6(3 c)$ & $8.7 \pm 5.1(4 c)$ & $4.7 \pm 1.6$ & $5.4 \pm 2.1$ \\
\hline LH/FSH ratio & $2.7 \pm 1.0(1 \mathrm{a}-\mathrm{e})$ & $1.1 \pm 0.65(2 b, d)$ & $2.5 \pm 1.1(3 c-e)$ & $1.8 \pm 0.82(4 c, e)$ & $1.1 \pm 0.33$ & $0.96 \pm 0.39$ \\
\hline Testosterone (nmol/l) & $2.85 \pm 0.8(1 \mathrm{a}, \mathrm{c}-\mathrm{e})$ & $2.4 \pm 1.2(2 \mathrm{c}-\mathrm{e})$ & $2.76 \pm 1.0(3 \mathrm{c}-\mathrm{e})$ & $1.99 \pm 0.89(4 c, e)$ & $1.37 \pm 0.46$ & 1.37 \\
\hline SHBG $(\mathrm{nmol} / \mathrm{l})$ & $45.2 \pm 21.7(1 a, b, d)$ & $26.9 \pm 10.1(2 b-e)$ & $22.5 \pm 11.4(3 \mathrm{c}, \mathrm{e})$ & $19.8 \pm 10.69(4 \mathrm{c}, \mathrm{e})$ & $38.0 \pm 13.6$ & $51.1 \pm 14.4$ \\
\hline FAI & $8.04 \pm 4.7(1 b-e)$ & $9.3 \pm 3.9(2 b-e)$ & $15.2 \pm 9.8(3 \mathrm{c}, \mathrm{e})$ & $11.46 \pm 4.5(4 \mathrm{c}, \mathrm{e})$ & $4.0 \pm 1.7$ & $2.9 \pm 1.3$ \\
\hline \multicolumn{6}{|l|}{ 17-OH-Prog (nmol/l) } & $3.95 \pm 1.61$ \\
\hline $\mathrm{Oh}$ & $5.5 \pm 1.8(1 \mathrm{a}, \mathrm{c}-\mathrm{e})$ & $10.9 \pm 36(2 b-e)$ & $4.7 \pm 1.8(3 \mathrm{c}, \mathrm{e})$ & $3.9 \pm 2.1(4 \mathrm{c})$ & $2.7 \pm 0.9$ & $2.7 \pm 1.1$ \\
\hline $1 \mathrm{~h}$ & $8.2 \pm 2.7(1 \mathrm{c})$ & $22 \pm 63(2 \mathrm{c}, \mathrm{e})$ & $9.4 \pm 4.8(3 \mathrm{c}, \mathrm{e})$ & $7.9 \pm 2.4(4 \mathrm{c})$ & $6.7 \pm 2.1$ & $5.7 \pm 1.8$ \\
\hline EPO (\%) & $100(1 \mathrm{a}, \mathrm{c}-\mathrm{e})$ & $22.2(2 \mathrm{e})$ & $100(3 c-e)$ & $18.75(4 \mathrm{e})$ & 54.6 & $20-$ \\
\hline \multicolumn{7}{|l|}{ Glucose $(\mathrm{nmol} / \mathrm{l})$} \\
\hline $\mathrm{Oh}$ & $4.7 \pm 0.4$ & $4.6 \pm 0.6$ & $4.9 \pm 0.8(3 c)$ & $4.7 \pm 0.3$ & $4.7 \pm 0.4$ & $4.5 \pm 0.3$ \\
\hline $1 \mathrm{~h}$ & $5.6 \pm 1.6(1 b, c)$ & $6.5 \pm 2.1(2 \mathrm{c})$ & $7.2 \pm 2.5(3 c, e)$ & $6.5 \pm 1.7(4 \mathrm{c})$ & $4.9 \pm 1.9$ & $3.5 \pm 0.7$ \\
\hline $2 \mathrm{~h}$ & $4.8 \pm 1.2(1 \mathrm{~b}, \mathrm{c}, \mathrm{d})$ & $4.9 \pm 0.98(2 b, d)$ & $5.6 \pm 1.8(3 \mathrm{c})$ & $5.7 \pm 0.9(4 c)$ & $4.9 \pm 1.9$ & $4.7 \pm 1.2$ \\
\hline \multicolumn{7}{|l|}{ Insulin (mU/l) } \\
\hline $\mathrm{Oh}$ & $5.7 \pm 2.1(1 a, b, d)$ & $9.7 \pm 7.79(2 b, c)$ & $16 \pm 16(3 \mathrm{c}, e)$ & $13.2 \pm 12(4 c)$ & $6.5 \pm 3.1$ & $5.2 \pm 2.8$ \\
\hline $1 \mathrm{~h}$ & $50.4 \pm 20(1 a-d)$ & $89 \pm 84(2 b, c)$ & $137 \pm 104(3 \mathrm{c}, \mathrm{e})$ & $117 \pm 96(4 \mathrm{c}, \mathrm{e})$ & $48.3 \pm 23$ & $31.2 \pm 18.2$ \\
\hline $2 \mathrm{~h}$ & $30.2 \pm 18(1 \mathrm{a}, \mathrm{b}, \mathrm{d})$ & $65 \pm 92(2 b-d)$ & $124 \pm 155(3 c, e)$ & $92 \pm 61(4 \mathrm{c})$ & $42.0 \pm 18(5)$ & $26.7 \pm 15.1$ \\
\hline HOMA-IR index* & $1.2 \pm 0.5(1 \mathrm{a}, \mathrm{b}, \mathrm{d})$ & $2.1 \pm 1.8(2 \mathrm{~b}, \mathrm{c})$ & $3.6 \pm 3.9(3 \mathrm{c}, \mathrm{e})$ & $2.7 \pm 2.3(4 c)$ & $1.4 \pm 0.8$ & $1.0 \pm 0.6$ \\
\hline $\mathrm{BMI}\left(\mathrm{kg} / \mathrm{m}^{2}\right)$ & $21.8 \pm 2.0(1 a, b, d)$ & $26.9 \pm 7.0(2 b, c)$ & $30.7 \pm 6.2(3 \mathrm{c}, \mathrm{e})$ & $28.7 \pm 5.7(4 c)$ & $23.8 \pm 3.1(5)$ & $21.1 \pm 1.8$ \\
\hline
\end{tabular}

$P$ values (given in brackets) ranged from $<0.05$ to $<0.0001$ and were derived from Wilcoxon rank sum test and Fisher's two-sided exact test (EPO). FCA functional cutaneous androgenization; FAS, functional androgenization syndrome; AMH, anti-Müllerian hormone; SHBG, sex hormone-binding globulin; FAI, free androgen index calculated as: testosterone/SHBG $\times 100$; 17-OH-Prog, 17-hydroxy-progesterone; EPO, enlarged polyfollicular ovaries; HOMA-IR, homeostasis model assessment of insulin resistance; BMI, body mass index. * (Insulin $0 \mathrm{~h}) \times($ Glucose $0 \mathrm{~h}) / 22.5$. Description of significant differences between groups: 1: FAS I vs other groups (1a: FAS II; 1b: FAS III; 1c: control; 1d: FAS IV; 1e: FCA). 2: FAS II vs other groups (2b: FAS III; 2c: control; 2d: FAS IV; 2e: FCA). 3: FAS III vs other groups (3c: control; 3d: FAS IV; 3e: FCA). 4: FAS IV vs other groups (4c: control; 4e: FCA). 5: FCA vs control. 